\title{
Investigation of photophysical, structural aspects and nonlinear optical properties of Foron blue SR analogs using Density Functional Theory (DFT)
}

\author{
ARCHANA A BHAGWAT and NAGAIYAN SEKAR* \\ Department of Dyestuff Technology, Institute of Chemical Technology, Matunga, Mumbai, Maharashtra \\ 400 019, India \\ E-mail: nethi.sekar@gmail.com
}

MS received 30 June 2018; revised 4 April 2019; accepted 11 April 2019; published online 21 June 2019

\begin{abstract}
Analogs of 2-[(2Z)-2-[[4-(dihexylamino)-2-methylphenyl]methylidene]-1,1-dioxo-1-benzothiophen-3-ylidene]propanedinitrile (Foron blue SR, Disperse Blue 354) are studied in terms of photophysical, structural aspects and its nonlinear optical properties evaluated by using Density Functional Theory (DFT) and Time Dependent Density Functional Theory (TD-DFT). The structures were optimized by using function B3LYP and basis set used was 6-31G(d). Polarizability $(\alpha)$, first and second order hyperpolarizability $(\beta$ and $\gamma$ ) was determined by three density functionals B3LYP, CAM-B3LYP and BHandHLYP. B3LYP functional and the basis set $6-311+\mathrm{G}(\mathrm{d}, \mathrm{p})$ shows high values of $\alpha, \beta$ and $\gamma$. The B3LYP functional gives the highest first order hyperpolarizability value for (Z)-2-(2-(4-(dimethylamino)benzylidene)-1,1dioxidobenzo[b]thiophen-3(2H)-ylidene)malononitrile $(\mathbf{3 a})$ is $373.31 \times 10^{-30}$ e.s.u and $486.32 \times 10^{-30}$ e.s.u in ethylacetate and dimethylformamide respectively. Effect of bond length alteration (BLA) and bond order alteration (BOA) on first order hyperpolarizability was evaluated. Intrinsic hyperpolarizability shows the compound (Z)-2-(4-(dimethylamino)benzylidene)benzo[b]thiophen-3(2H)-one 1,1-dioxide (1a) intrinsically good. Perturbation potential is found to decrease as absorption energy deceases and hyperpolarizability increases.
\end{abstract}

Keywords. DFT; BLA; BOA; NLO; first and second order hyperpolarizability.

\section{Introduction}

Nonlinear optics and nonlinear optical (NLO) materials are the growing areas in the light of the fact these materials have applications in optoelectronics, ${ }^{1,2}$ data storage and fiber optic communication. ${ }^{3,4}$ Traditionally various inorganic compounds like potassium dihydrogen phosphate, potassium titanyl phosphate, Neodymium Yttrium Aluminium Garnate were studied as NLO materials. In recent years organic molecules are becoming important as NLO materials. ${ }^{5,6}$ One of the ideal candidate as organic NLOphores are the push pull dyes which contain donor, acceptor and $\pi$-bridge. Such molecules show high charge transfer (CT) characteristics and consequently high dipole moments and polarizabilities. ${ }^{7}$ The NLO properties of the molecules can be determined experimentally by standard methods like Electric Field Induced Second Harmonic Generation (EFISHG) ${ }^{8,9}$ or Hyper Rayleigh scattering (HRS). ${ }^{10,11}$ In recent years, solvatochromic methods ${ }^{12}$ and computational methods are gaining importance in obtaining NLO properties. Both these methods particularly computational methods serve as a priori tool in understanding trends in NLO properties of organic molecules without much recourse to expensive and elaborate experimental methods. ${ }^{13,14}$

Various density functionals like B3LYP, PBE0, HF, LC-B3LYP, CAM-B3LYP are used for NLO computations, and among all the range separated hybrid functional, CAM-B3LYP gives better results. ${ }^{15}$ So we have selected B3LYP, CAM-B3LYP, BHandHLYP functionals to compute the hyperpolarizability values for selected compounds.

\footnotetext{
*For correspondence

Electronic supplementary material: The online version of this article (https://doi.org/10.1007/s12039-019-1632-7) contains supplementary material, which is available to authorized users.
} 
<smiles>CN(C)c1ccc(/C=C2/C(=O)c3ccccc3S2(=O)=O)cc1</smiles>

$1 \mathrm{a}$

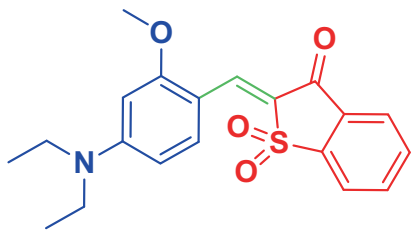

$1 \mathrm{~b}$

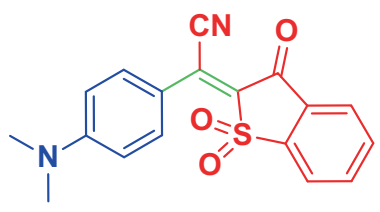

$2 a$

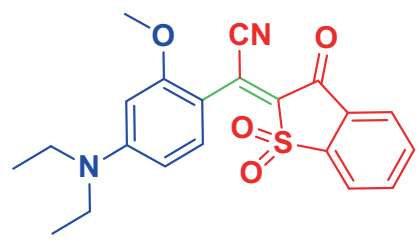

$2 b$<smiles>CN(C)c1ccc(/C=C2\C(=C(C#N)C#N)c3ccccc3S2(=O)=O)cc1</smiles>

$3 a$

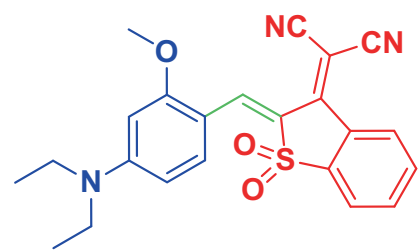

$3 b$

Figure 1. Structure of the compounds $\mathbf{1 a}, \mathbf{2 a}, \mathbf{3 a}, \mathbf{1 b}, \mathbf{2 b}$ and $\mathbf{3 b}$.

Oudar proposed a two-level model which established structure-NLO properties relationship. This model helps to choose donor and acceptor in order to obtain maximum hyperpolarizability. ${ }^{16,17}$ Solvent dependant hyperpolarizabilities can be determined by the Oudars equation. Compounds having a large difference in dipole moments between the ground and excited states, a large value of transition dipole moment and a red shifted absorption particularly beyond $600 \mathrm{~nm}$ exhibit large values for linear polarizability $(\alpha)$, hyperpolarizability $\left(\beta_{0}\right)$, and second order hyperpolarizability $(\gamma)$.

Molecules selected for Time Dependant Density Functional Theory (TD-DFT) study are non-centro symmetric having uneven distribution of donors and acceptors and so will satisfy the all requirements. As push pull dyes have reasonably higher value as NLO materials we have selected polymethine dyes which contain electron donor and acceptor group linked by $\pi$ - bridge for TD-DFT study. Nowadays Density Functional Theory (DFT) and TD-DFT is a popular tool to study structural, molecular and electronic also photophysical properties thoroughly. ${ }^{18}$

Here we have studied styryl compounds derived from 3-oxo-2,3-dihydrobenzo-[b]thiophene-1,1-dioxide (compounds $\mathbf{1 a}$ and $\mathbf{1 b}$ ) and, its derivatives obtained with oxidative cyanation (compound $\mathbf{2 a}$ and $\mathbf{2 b}$ ), and 3dicyanomethylene-2,3dihydrobenzo[b]thiophene-1,1dioxide (compound 3a and 3b) (Figure 1) ${ }^{19}$ by using Density Functional Theory [B3LYP/6-31G(d)] and Time Dependent Density Functional Theory [TD-B3LYP/6-31G(d)]. The structures were optimized by using function B3LYP and basis set used was 6$31 \mathrm{G}(\mathrm{d})$. Incorporation of cyano group into styryl dye gives a red shift in absorption. Experimentally observed photophysical properties of compound $\mathbf{1 a}, \mathbf{1 b}, \mathbf{2 a}$, $\mathbf{2 b}, \mathbf{3 a}$ and $\mathbf{3 b}$ are compared with vertical excitations obtained from TD-DFT computations. Linear and nonlinear behaviour of the compounds were studied in terms of estimation of polarizability $(\alpha)$, first and second order hyperpolarizability $\left(\beta_{0}\right.$ and $\left.\gamma\right)$ by three different density functionals viz. B3LYP, CAM-B3LYP and BHandHLYP.

\section{Materials and methods}

\subsection{Computation strategy}

Gaussian 09 program package was used to perform all the DFT and TD-DFT computations. ${ }^{20}$ The ground state $\left(\mathrm{S}_{0}\right)$ geometry of the dyes was optimized in the gas phase using DFT. ${ }^{21}$ The functional used was B3LYP. The B3LYP method combines Becke's three parameter exchange functional (B3) ${ }^{22}$ with the nonlocal correlation functional by Lee, Yang and Parr (LYP). ${ }^{23}$ The basis set used for all the atoms was $6-31 G(d)$. The vertical excitation energies and oscillator strength were obtained by using the TD-DFT using the same hybrid functional and basis set. ${ }^{24,25}$ The low-lying first singlet excited states $\left(\mathrm{S}_{1}\right)$ of all the compounds were relaxed to obtain their minimum energy geometry. The vertical emissions were calculated using the TD-DFT method with the optimized first singlet excited state geometry. The Polarizable Continuum Model (PCM) as implemented in Gaussian 09 was used to optimize the ground and excited state geometries in dimethylformamide and ethyl acetate. All polar computation done by using three functionals viz., B3LYP, CAM-B3LYP and BHandHLYP. 
Table 1. Selected bond lengths, bond angles and dihedral angles of 1a and 1b, using B3LYP 6-31G(d), (bond lengths are in $\AA$, bond angles and dihedral angles are in degree).

\begin{tabular}{|c|c|c|c|c|c|c|c|}
\hline \multirow[b]{3}{*}{ Atom number } & \multicolumn{3}{|c|}{$1 \mathbf{a}$} & \multirow[b]{3}{*}{ Atomnumber } & \multicolumn{3}{|c|}{$\mathbf{1 b}$} \\
\hline & \multirow{2}{*}{$\begin{array}{c}\text { Gas } \\
\text { GS }\end{array}$} & \multicolumn{2}{|c|}{ DMF } & & \multirow{2}{*}{$\begin{array}{c}\text { Gas } \\
\text { GS }\end{array}$} & \multicolumn{2}{|c|}{ DMF } \\
\hline & & GS & ES & & & GS & ES \\
\hline Bond length & & & & Bond length & & & \\
\hline $\mathrm{N}_{28}-\mathrm{C}_{18}$ & 1.37 & 1.36 & 1.36 & $\mathrm{~N}_{27}-\mathrm{C}_{18}$ & 1.38 & 1.37 & 1.36 \\
\hline $\mathrm{C}_{21}-\mathrm{C}_{17}$ & 1.44 & 1.43 & 1.48 & $\mathrm{C}_{21}-\mathrm{C}_{17}$ & 1.43 & 1.42 & 1.47 \\
\hline $\mathrm{C}_{17}-\mathrm{C}_{7}$ & 1.36 & 1.37 & 1.38 & $\mathrm{C}_{17}-\mathrm{C}_{7}$ & 1.36 & 1.37 & 1.38 \\
\hline Bond angle & & & & Bond angle & & & \\
\hline $\mathrm{C}_{20}-\mathrm{C}_{21}-\mathrm{C}_{17}$ & 133.68 & 134.27 & 126.09 & $\mathrm{C}_{20}-\mathrm{C}_{21}-\mathrm{C}_{17}$ & 133.05 & 133.9 & 126.11 \\
\hline $\mathrm{C}_{17}-\mathrm{C}_{7}-\mathrm{C}_{8}$ & 120.88 & 121.22 & 126.61 & $\mathrm{C}_{17}-\mathrm{C}_{7}-\mathrm{C}_{8}$ & 120.78 & 121.04 & 126.55 \\
\hline $\mathrm{C}_{17}-\mathrm{C}_{7}-S_{14}$ & 128.82 & 128.69 & 123.16 & $\mathrm{C}_{17}-\mathrm{C}_{7}-S_{14}$ & 128.93 & 128.8 & 123.19 \\
\hline Dihedral angle & & & & Dihedral angle & & & \\
\hline $\mathrm{C}_{21}-\mathrm{C}_{17}-\mathrm{C}_{7}-\mathrm{S}_{14}$ & 4.7 & 5.08 & 0.34 & $\mathrm{C}_{21}-\mathrm{C}_{17}-\mathrm{C}_{7}-\mathrm{S}_{14}$ & 6 & 6.7 & 3.65 \\
\hline $\mathrm{C}_{22}-\mathrm{C}_{21}-\mathrm{C}_{17}-\mathrm{C}_{7}$ & 10.66 & 8.95 & 90.35 & $\mathrm{C}_{22}-\mathrm{C}_{21}-\mathrm{C}_{17}-\mathrm{C}_{7}$ & 13.3 & 11.07 & 90.58 \\
\hline
\end{tabular}

\section{Results and Discussion}

\subsection{Geometrical parameters}

Optimization of the structure of $\mathbf{1 a}, \mathbf{1} \mathbf{b}, \mathbf{2 a}, \mathbf{2} \mathbf{b}, \mathbf{3 a}$ and 3b was done by using TD-B3LYP/6-31G(d) method and results are presented in Table 1 for representative example 1a and $\mathbf{1 b}$. The change in molecules from ground state to excited state is shown in terms of bond length, bond angle and dihedral angles of the molecules.

The optimized structures (Figure S1, Supplementary Information) of the compounds show that the two aromatic rings i.e., donor and acceptor are planar in ground state and slightly twisted in the excited state due to steric hindrance. This is supported by dihedral angles in the excited state geometry. Dihedral angles $\left(\mathrm{C}_{5}-\mathrm{C}_{4}-\mathrm{C}_{7}-\mathrm{C}_{8}\right)$ of $\mathbf{1 a}$ and $\mathbf{1 b}$ are $90.35^{\circ}$ and $90.58^{\circ}$, in $\mathbf{2 a}$ and $\mathbf{2 b}$ are $90.67^{\circ}$ and $90.92^{\circ}$ and in $\mathbf{3 a}$ and $\mathbf{3 b}$ are $91.50^{\circ}$ and $91.35^{\circ}$ respectively in DMF at the first excited state.

The ground state optimized geometry shows planarity with slight twist $8^{\circ}-20^{\circ}$ in the molecule with a small dihedral angle for $\mathbf{1 a}, \mathbf{1 b}$ and $\mathbf{3 a}, \mathbf{3 b}$. Due to cyano group at $\mathrm{C}_{8}$ position, dihedral angle becomes $40-43^{\circ}$ in $\mathbf{2 a}$ and $\mathbf{2 b}$ (Table S1-S5 and optimized structures of 1a, 1b, 2a, $\mathbf{2 b}$ and 3a in Figure S1, Supplementary Information).

\subsection{Vertical excitation and oscillator strength}

To investigate vertical excitations of the compounds TD-B3LYP method was used. Vertical excitation, oscillator strength, orbital major contribution are reported in Table 2 for N,N-Dimethylformamide (DMF) and ethylacetate (EA) solvents. To compare experimental absorption with vertical excitation these two solvents were chosen because experimental absorption values in DMF and EA are available in the literature. ${ }^{19}$

From Table 2, it is observed that vertical excitations are in the range of the experimentally obtained absorption values with small $\%$ deviation. In all the compounds, an electronic transition occurs from Highest Occupied Molecular Orbital (HOMO) to Lowest Occupied Molecular Orbital (LUMO). The trend in vertical excitation was found as series $\mathbf{1}<$ series $\mathbf{2}<$ series 3 . This is because of the strength of acceptor increases due to cyano group in series $\mathbf{2}$ and $\mathbf{3}$. Compounds 1a, $\mathbf{2 a}$ and 3a absorb at $438 \mathrm{~nm}, 506 \mathrm{~nm}$ and $533 \mathrm{~nm}$ respectively in N,N-Dimethylformamide (DMF). The same trend was observed in ethyl acetate. Bathochromic shift was observed in DMF as compared to ethyl acetate, which suggests positive solvatochromism. Oscillator strength was found to be within $0.6-1.0$.

\subsection{Prediction of the dipole moment}

The dipole moment is an important factor to understand polarization of the molecule. It is evaluated in the ground state and excited state.

Computed dipole moment shows that at ground state, dipole moment is higher (Table 3) than at excited state, which indicates that charge separation is more in the ground state due to planar geometry while in excited state geometry it is orthogonal, and hence charge separation is less and consequently dipole moment decreased in an excited state. The trend observed in dipole moment values as series $\mathbf{1}<$ series $\mathbf{2}<$ series $\mathbf{3}$. Compound 3b shows the largest dipole moment. The strength of donor and acceptor enhances the dipole moment. More the charge separation, larger is the dipole moment (from 
Table 2. Optical properties of the compounds.

\begin{tabular}{|c|c|c|c|c|c|c|c|c|c|}
\hline & Solvent & $\lambda \operatorname{abs}^{a}(\mathrm{~nm})$ & $\lambda \operatorname{abs}^{b}$ (theo) (nm) & $\lambda \operatorname{abs}^{c}$ Gas & $f^{d}$ & $D^{e}$ & Major contribution & $\%$ & $\mathrm{H}-\mathrm{L}$ \\
\hline \multirow[t]{2}{*}{$1 \mathbf{a}$} & DMF & 488 & 438 & 404 & 0.9872 & 10.24 & 0.70314 & 98.88 & $82->83$ \\
\hline & EA & 473 & 433 & & 0.9534 & 8.45 & 0.70301 & 98.84 & $82->83$ \\
\hline \multirow[t]{2}{*}{$1 b$} & DMF & 500 & 438 & 407 & 1.048 & 12.4 & 0.70243 & 98.68 & $98->99$ \\
\hline & EA & 484 & 433 & & 1.0094 & 10.53 & 0.70227 & 98.64 & $98->99$ \\
\hline \multirow[t]{2}{*}{$2 \mathbf{a}$} & $\mathrm{DMF}$ & 560 & 506 & 461 & 0.8418 & 9.82 & 0.70814 & 100.29 & $88->89$ \\
\hline & EA & 540 & 499 & & 0.8055 & 7.59 & 0.70765 & 100.15 & $88->89$ \\
\hline \multirow[t]{2}{*}{$2 b$} & DMF & 580 & 536 & 496 & 0.6753 & 7.58 & 0.70575 & 99.62 & $104->105$ \\
\hline & EA & 552 & 530 & & 0.6301 & 3.98 & 0.70482 & 99.35 & $104->105$ \\
\hline \multirow[t]{2}{*}{$3 \mathbf{a}$} & DMF & 592 & 534 & 492 & 0.8918 & 9.79 & 0.70672 & 99.89 & $94->95$ \\
\hline & EA & 570 & 528 & & 0.8589 & 7.36 & 0.7066 & 99.86 & $94->95$ \\
\hline \multirow[t]{2}{*}{$3 b$} & DMF & 608 & 532 & 495 & 0.9368 & 12.5 & 0.70642 & 99.81 & $110->111$ \\
\hline & EA & 596 & 527 & & 0.8976 & 11.57 & 0.70629 & 99.77 & $110->111$ \\
\hline
\end{tabular}

a Experimental absorption

$b$ Vertical excitation (theoretical)

${ }^{c}$ vertical excitation in gas phase

$d$ Oscillator strength

$e \%$ deviation.

Table 3. Computed ground and excited state dipole moments of the compounds in vacuum and solvents using B3LYP/6$31 \mathrm{G}(\mathrm{d})$.

\begin{tabular}{|c|c|c|c|c|c|c|c|c|c|c|}
\hline \multirow[t]{2}{*}{ Compound } & \multicolumn{4}{|c|}{$\mu(\mathrm{DMF})$} & \multirow[b]{2}{*}{$\mu_{g}^{b}-\mu_{g}^{a}$} & \multicolumn{4}{|c|}{$\mu(\mathrm{EA})$} & \multirow[b]{2}{*}{$\mu_{g}^{b}-\mu_{g}^{a}$} \\
\hline & $\mu_{g}^{a}$ & $\mu_{g}^{b}$ & $\mu_{e}^{c}$ & $\mu_{g}^{b-} \mu_{e}^{c}$ & & $\mu_{g}^{a}$ & $\mu_{g}^{b}$ & $\mu_{e}^{c}$ & $\mu_{g}^{b-} \mu_{e}^{c}$ & \\
\hline $1 \mathbf{a}$ & 4.1 & 6.3 & 1.1 & 5.2 & 2.2 & 4.1 & 5.8 & 1.2 & 4.6 & 1.7 \\
\hline $1 b$ & 5.8 & 8.8 & 2.8 & 6.0 & 3.0 & 5.8 & 8.1 & 2.7 & 5.4 & 2.3 \\
\hline $2 \mathbf{a}$ & 5.5 & 8.3 & 6.8 & 1.5 & 2.7 & 5.5 & 7.6 & 5.8 & 1.7 & 2.1 \\
\hline $2 b$ & 5.9 & 8.9 & 7.1 & 1.8 & 3.0 & 5.9 & 8.2 & 6.9 & 1.2 & 2.3 \\
\hline $3 \mathbf{a}$ & 8.0 & 11.5 & 5.8 & 5.7 & 3.5 & 8.0 & 10.8 & 5.8 & 5.0 & 2.8 \\
\hline 3b & 9.3 & 13.8 & 7.0 & 6.8 & 4.4 & 9.3 & 12.7 & 6.9 & 5.8 & 3.4 \\
\hline
\end{tabular}

${ }^{a}$ Dipole moment of the compounds in gas phase

${ }^{b}$ Dipole moment of the compounds in ground state

${ }^{c}$ Dipole moment of the compounds in excited state.

series 1 to 3). Dipole moment increases more in solvents than gas phase because of solvation. In the excited state geometry, more stabilisation of charge separated structures due to solvation may be responsible for the drastic decrease in the dipole moment. This trend correlated well with vertical excitation energies of the compounds. Dipole moment and vertical excitation energies are found higher in solvent phase than gas phase.

\subsection{Frontier molecular orbitals (FMO)}

Highest occupied molecular orbital (HOMO) and Lowest unoccupied molecular orbital (LUMO) of the compounds was studied to understand charge transfer characteristics in compounds from donor to acceptor in the ground state and excited state. From Figure 3 it is clear that in HOMO of the ground state electron density is located on donor part while in LUMO it slightly shifted towards sulphone ring. In HOMO of the excited state electron density at donor which is completely shifted towards sulphone ring in LUMO. Because of the cyano groups in series of compound $\mathbf{2}$ and $\mathbf{3}$, acceptor strength increases and decrease in LUMO level observed. HOMO-LUMO energy gap trend was found as $\mathbf{3 a}<\mathbf{3 b}<\mathbf{2} \mathbf{b}<\mathbf{2} \mathbf{a}<\mathbf{1 a}<\mathbf{1 b}$ (Figure 2). The strong acceptor 2-(1,1-dioxidobenzo[b]thiophen$3(2 \mathrm{H})$-ylidene)malononitrile, decreases energy gap in $3 a$.

\subsection{Nonlinear optical properties (NLO)}

Using finite field approach, we have calculated, the static dipole moment $(\mu)$, mean polarizability $\left(\alpha_{0}\right)$, polarizability anisotropy $(\Delta \alpha)$, static first order hyperpolarizability $\left(\beta_{0}\right)$ and second hyperpolarizability $(\gamma)^{26}$ for the compounds of series 1-3 using B3LYP/6-311+g(d,p), 

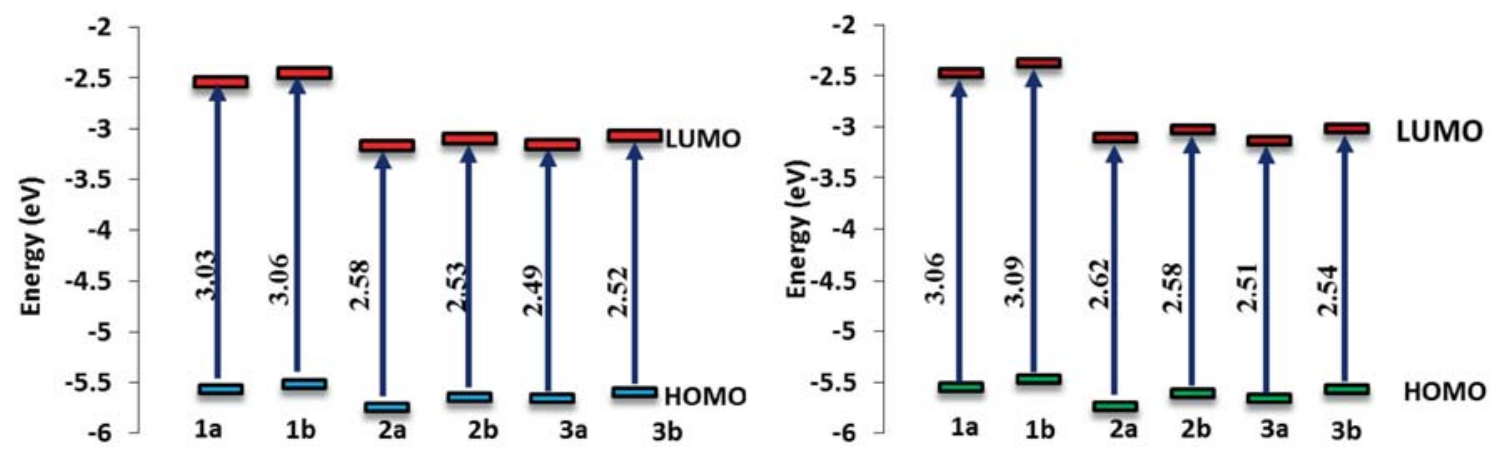

Figure 2. HOMO-LUMO energy gap in DMF (left) and ethyl acetate (right).
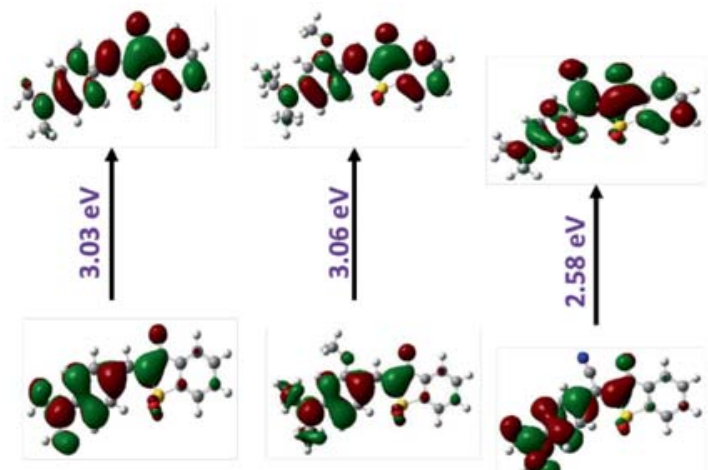

$1 a$

1b

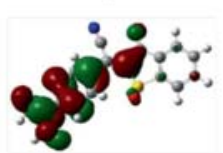

$2 a$

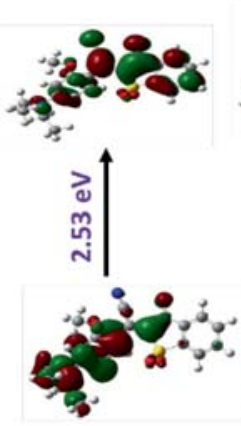

2b

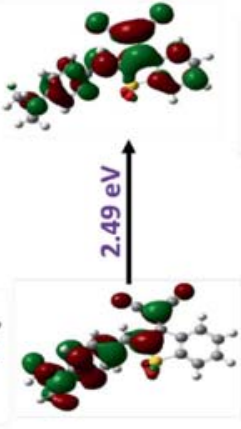

3a

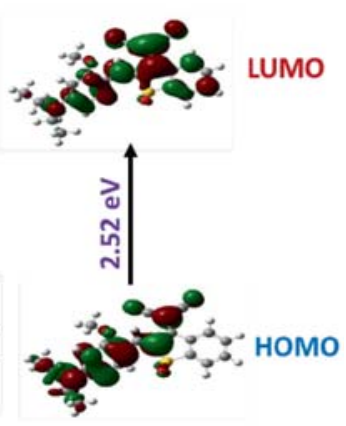

$3 b$

Figure 3. FMO diagram of compounds $\mathbf{1 a}, \mathbf{2} \mathbf{a}, \mathbf{3 a}, \mathbf{1 b}, \mathbf{2} \mathbf{b}$ and $\mathbf{3 b}$ in DMF at ground state.

Table 4. Linear polarizability $\alpha_{0}$, first order hyperpolarizability $\beta_{0}$ and second order hyperpolarizability $\gamma$ in ethylacetate.

\begin{tabular}{|c|c|c|c|c|c|c|c|c|c|}
\hline \multicolumn{4}{|c|}{$\alpha_{0}\left(10^{-24} \mathrm{esu}\right)$} & \multicolumn{3}{|c|}{$\beta_{0}\left(10^{-30} \mathrm{esu}\right)$} & \multicolumn{3}{|c|}{$\gamma\left(10^{-36} \mathrm{esu}\right)$} \\
\hline & B3LYP & CAM-B3LYP & BHandHLYP & B3LYP & CAM-B3LYP & BHandHLYP & B3LYP & CAM-B3LYP & BHandHLYP \\
\hline $1 \mathbf{a}$ & 59.51 & 55.77 & 54.5 & 232.9 & 191.18 & 174.92 & 480.81 & 351.87 & 340.86 \\
\hline $1 b$ & 67.71 & 63.84 & 62.13 & 211.32 & 172.06 & 156.86 & 467.63 & 303.56 & 304.99 \\
\hline $2 \mathbf{a}$ & 65.28 & 60.9 & 59.83 & 290.07 & 267.34 & 251.54 & 431.69 & 466.6 & 446.94 \\
\hline $2 \mathrm{~b}$ & 71.83 & 66.55 & 65.17 & 319.07 & 250.6 & 237.76 & 608.94 & 528.38 & 515.63 \\
\hline $3 \mathbf{a}$ & 77.46 & 72.59 & 70.98 & 373.31 & 349.92 & 317.28 & 537.79 & 577.85 & 569.05 \\
\hline $\mathbf{3 b}$ & 85.71 & 81.03 & 78.85 & 322.59 & 295.24 & 269.64 & 474.81 & 374.82 & 414.68 \\
\hline
\end{tabular}

BHandHLYP/6-311+g(d,p) and CAMB3LYP/6-311+ $\mathrm{g}(\mathrm{d}, \mathrm{p})$ method.

From Table 4 and S6 (Supplementary Information) it is revealed that $\alpha, \beta_{0}$ and $\gamma$ values are higher by B3LYP method. As the strength of donor and acceptor is more in the series of compounds $\mathbf{b}$, compounds $\mathbf{1 b}, \mathbf{2 b}$ and $\mathbf{3 b}$ show more linear polarizability than 1a, 2a and 3a respectively. Within the series, donor remains constant but the strength of acceptor increases, therefore, in comparison to $\mathbf{1 a}, \mathbf{2} \mathbf{a}$ and $\mathbf{3 a}$, an increasing trend of linear polarizabiliy was observed. The same trend is observed in the $\mathbf{1} \mathbf{b}-\mathbf{2} \mathbf{b}-\mathbf{3 b}$ series. The first order hyperpolarizability decreases from 1a to $\mathbf{1 b}, \mathbf{2 a}$ to $\mathbf{2 b}$ and $\mathbf{3 a}$ to $\mathbf{3 b}$ in all basis set except in $\mathbf{2 a}$ to $\mathbf{2 b}$ which show an increased value in B3LYP method. In 1a-2a-3a series, enhancement in first order hyperpolarizability is observed. The same was observed in $\mathbf{1 b}-\mathbf{2 b} \mathbf{b}-\mathbf{3 b}$ series. The second order hyperpolarizabilities observed in $\mathbf{1 b}$ and $\mathbf{2} \mathbf{b}$ are more than for $\mathbf{1 a}$ and 1b. The increased values were observed for 1a-2a-3a for B3LYP, CAM-B3LYP and BHandHLYP (Figure 4). As acceptor strength increases polarizability, first and second hyperpolarizability increase. From series $\mathbf{1}$ to $\mathbf{3}$ acceptor strength increases by one and two cyano groups 


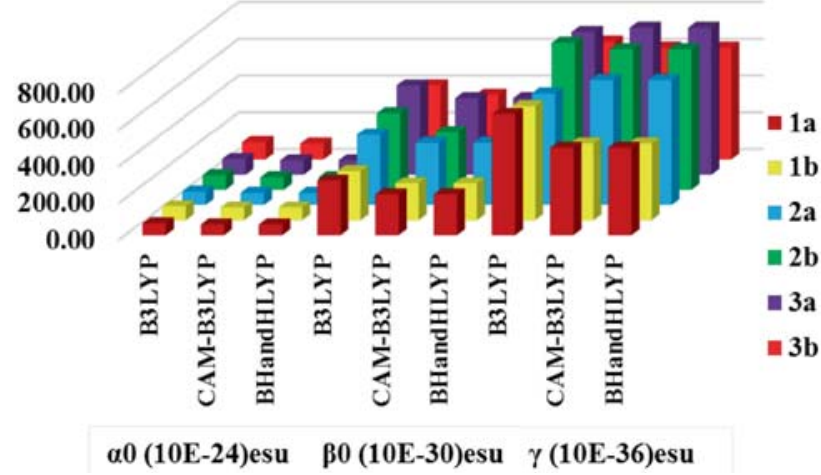

Figure 4. $\alpha_{0}, \beta_{0}$ and $\gamma$ values trend for $\mathbf{1 a}, \mathbf{1 b}, \mathbf{2 a}, \mathbf{2 b}$, $\mathbf{3 a}$ and $\mathbf{3 b}$ by density functionals B3LYP, CAM-B3LYP and BHandLYP.

respectively (Figure 1). Overall, it can be concluded that the strength of substituents - donor and acceptor - influences the linear and nonlinear optical properties.

\subsection{Fundamental limits for the hyperpolarizability value}

Kuzyk proposed a theory for fundamental limits of NLO response. ${ }^{27}$ It is a three-level model which gives two level limits (2L) for $\beta$. It is expressed as,

$\beta_{2 L}^{* S R} \leq \sqrt[4]{3}\left(\frac{n^{2}+2}{3}\right)^{3}\left(\frac{e \hbar}{\sqrt{m}}\right)^{3} \frac{N^{3 / 2}}{E_{10}^{7 / 2}}$

Where $e$ and $m$ are the charge and mass of the electron.

Equation (1) reveals that the limit depends on the refractive index $n$, the length of conjugation and number of electrons $N$ and transition energy $E_{10}$ between ground state and first excited state obtained from $\lambda$ max absorption.

The second order hyperpolarizability $(\gamma)$ limit is obtained from eq. (2)

$$
-\frac{e^{4} h^{4}}{m^{2}}\left(\frac{N^{2}}{E_{10}^{5}}\right) \leq \gamma \leq 4 \frac{e^{4} h^{4}}{m^{2}}\left(\frac{N^{2}}{E_{10}^{5}}\right)
$$

The values obtained from eqs. $(1,2)$, are summarized in Table 5 and compared with the value obtained from theoretical approach for compounds $\mathbf{1 a}-\mathbf{3 b}$. From Table 5, it was observed that the $\beta_{0}$ values are below the limit and $\gamma$ values are within the estimated limiting values for the compounds $\mathbf{1 a}-\mathbf{3 b}$.

\subsection{Intrinsic hyperpolarizability}

Intrinsic hyperpolarizability is an important aspect to determine the origin of the NLO response. This method is reliable to know the usefulness of the materials in a particular device. Therefore we have calculated the
Table 5. Fundamental limits of the hyperpolarizability.

\begin{tabular}{llccc}
\hline & & $\beta_{0}^{\max }$ & $\gamma_{\min }$ & $\gamma_{\max }$ \\
\cline { 3 - 5 } Compound & Solvent & $\left(10^{-30}\right.$ esu $)$ & $\left(10^{-36}\right.$ esu $)$ & $\left(10^{-36}\right.$ esu $)$ \\
\hline $\mathbf{1 a}$ & EA & 2005 & -3869 & 15477 \\
& DMF & 2530 & -4523 & 18092 \\
$\mathbf{1 b}$ & EA & 2173 & -4340 & 17363 \\
& DMF & 2755 & -5107 & 20429 \\
$\mathbf{2 a}$ & EA & 3188 & -7504 & 30017 \\
& DMF & 4096 & -9000 & 36003 \\
$\mathbf{2 b}$ & EA & 3443 & -8376 & 33504 \\
& DMF & 4631 & -10727 & 42908 \\
3a & EA & 3853 & -9833 & 39335 \\
& DMF & 4976 & -11883 & 47535 \\
3b & EA & 4504 & -12290 & 49162 \\
& DMF & 5462 & -13578 & 54315 \\
\hline
\end{tabular}

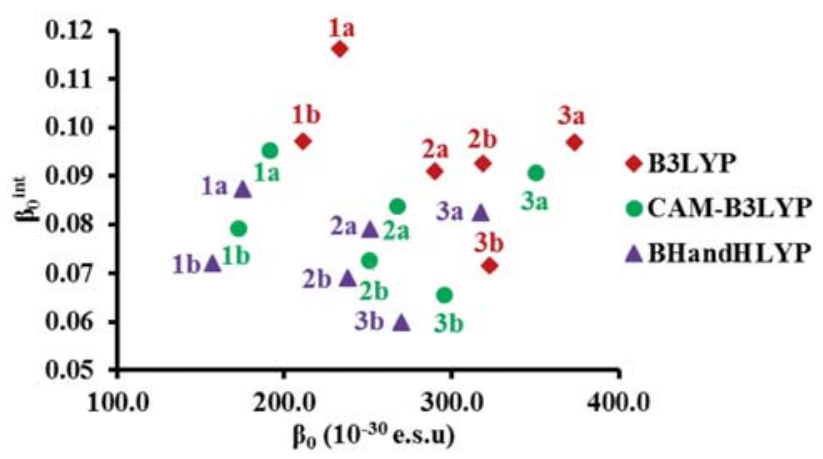

Figure 5. Plot of the first order hyperpolarizability $\beta_{0}$ and intrinsic hyperpolarizability $\beta_{0}^{\text {int }}$.

intrinsic hyperpolarizability of the compounds which is the measure of the ratio of hyperpolarizability and fundamental limit to the hyperpolarizability (eq. 3). ${ }^{28}$

$\beta_{0}^{\text {int }}=\frac{\beta_{0}}{\beta_{0}^{\max }}$

Where, $\beta_{0}$ and $\beta_{0}^{\max }$ are the first hyperpolarizability and fundamental limits to the first hyperpolarizability.

Table S7 and S8 (Supplementary Information) show the same trend of $\beta_{o}^{\text {int }}$ in DMF and EA. Series a shows larger $\beta_{0}$ and $\beta_{o}^{\text {int }}$ values than series $\mathbf{b}$. The trend of $\beta_{o}^{\text {int }}$ is observed as B3LYP $>$ CAM-B3LYP $>$ BhandHLYP in EA. Maximum intrinsic hyperpolarizability found for 1a is 0.11 . Therefore, it can be concluded that $\mathbf{1 a}$ is intrinsically good (Figure 5).

\subsection{Electrophilicity index}

To understand the chemical reactivity of the compounds, chemical hardness $(\eta)$ and global electrophilicity index $(\omega)^{29}$ were calculated as below, 
Table 6. Chemical potential, chemical hardness and electrophilicity index of 1a-3b in DMF optimized at B3LYP/6-31G(d) level.

\begin{tabular}{lcccccccc}
\hline Compound & HOMO & LUMO & $\mu \mathrm{eV}$ & $\eta \mathrm{eV}$ & $\omega \mathrm{eV}$ & $\omega^{+} \mathrm{eV}$ & $\omega^{-} \mathrm{eV}$ & $\omega^{ \pm} \mathrm{eV}$ \\
\hline $\mathbf{1 a}$ & -0.2046 & -0.0932 & -4.052 & 3.032 & 2.708 & 1.061 & 5.112 & 6.173 \\
$\mathbf{1 b}$ & -0.2028 & -0.09025 & -3.987 & 3.063 & 2.595 & 0.984 & 4.972 & 5.956 \\
$\mathbf{2 a}$ & -0.2112 & -0.11635 & -4.456 & 2.581 & 3.847 & 1.942 & 6.398 & 8.340 \\
$\mathbf{2 b}$ & -0.2073 & -0.114 & -4.372 & 2.539 & 3.763 & 1.895 & 6.266 & 8.161 \\
$\mathbf{3 a}$ & -0.2079 & -0.1162 & -4.410 & 2.495 & 3.896 & 2.003 & 6.413 & 8.416 \\
$\mathbf{3 b}$ & -0.2056 & -0.11265 & -4.330 & 2.529 & 3.706 & 1.857 & 6.187 & 8.045 \\
\hline
\end{tabular}

Table 7. Electrophilicity index of 1a-3b in EA optimized at B3LYP/6-31G(d) level.

\begin{tabular}{lcccccccc}
\hline Compound & HOMO & LUMO & $\mu \mathrm{eV}$ & $\eta \mathrm{eV}$ & $\omega \mathrm{eV}$ & $\omega^{+} \mathrm{eV}$ & $\omega^{-} \mathrm{eV}$ & $\omega^{ \pm} \mathrm{eV}$ \\
\hline $\mathbf{1 a}$ & -0.2038 & -0.09097 & -4.010 & 3.069 & 2.619 & 0.998 & 5.008 & 6.006 \\
$\mathbf{1 b}$ & -0.2011 & -0.08719 & -3.922 & 3.098 & 2.482 & 0.908 & 4.830 & 5.738 \\
$\mathbf{2 a}$ & -0.2108 & -0.11424 & -4.422 & 2.626 & 3.722 & 1.840 & 6.261 & 8.101 \\
$\mathbf{2 b}$ & -0.2060 & -0.11104 & -4.313 & 2.584 & 3.600 & 1.766 & 6.080 & 7.846 \\
$\mathbf{3 a}$ & -0.2079 & -0.11532 & -4.397 & 2.518 & 3.839 & 1.955 & 6.352 & 8.307 \\
$\mathbf{3 b}$ & -0.2045 & -0.11085 & -4.290 & 2.547 & 3.612 & 1.786 & 6.076 & 7.861 \\
\hline
\end{tabular}

$\mathrm{E}_{\mathrm{HOMO}}=$ Energy of the HOMO

$\mathrm{E}_{\mathrm{LUMO}}=$ Energy of the LUMO

$\mu=$ Chemical potential

$\eta=$ Chemical Hardness

$\omega=$ Electrophilicity Index

$\omega^{+}=$Electron accepting ability

$\omega^{-}=$Electron donating ability

$\omega^{ \pm}=$Net Electrophilicity index.

$\omega=\frac{\mu^{2}}{2 \eta}$

Where $\mu$ is the negative of the electronegativity

$\mu=-\frac{I+A}{2}$.

and $\eta$ is chemical hardness,

$\eta=I-A$

Where, $I$ is the electron potential $(I P)$ and $A$ is the electron affinity $(E A)$, By Koopmans theorem, $I P$ indicated as negative energy of the Highest Occupied Molecular Orbital (HOMO) and $E A$ as the negative energy of the Lowest Unoccupied Molecular Orbital (LUMO).

Energies of the HOMO and LUMO of the compounds were obtained from optimized geometries of the compounds by DFT method.

$\begin{aligned} \mu & =\frac{E_{\text {НОМО }}+E_{L U M O}}{2} \\ \eta & =E_{L U M O}-E_{\text {НОМO }}\end{aligned}$

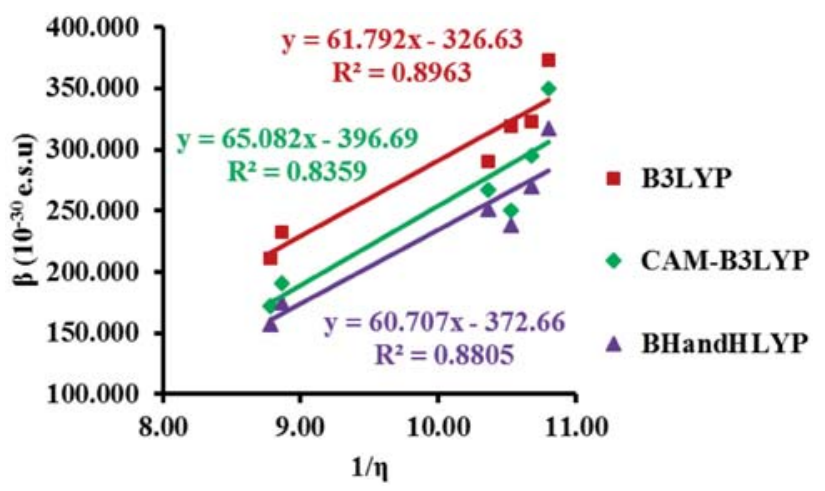

Figure 6. Plot of the inverse of chemical hardness and first order hyperpolarizability.

Therefore, to predict stabilities of the compounds, net electrophilicity index $\left(\omega^{ \pm}\right)$was calculated by using formula (9),

$\omega^{ \pm}=\omega^{+}+\omega^{-}$

Where $\omega^{+}$electron accepting power and $\omega^{-}$electron donating power were obtained from following equations.

$\omega^{+}=\frac{\left(E_{L U M O}\right)^{2}}{2\left(E_{L U M O}-E_{\text {HOMO }}\right)}$ 
Table 8. BLA and BOA of the compounds $\mathbf{1 a}, \mathbf{1 b}, \mathbf{2} \mathbf{a}, \mathbf{2 b}, \mathbf{3} \mathbf{a}$ and $\mathbf{3 b}$.

\begin{tabular}{cccccccc}
\hline & & $\mathbf{1 a}$ & $\mathbf{1 b}$ & $\mathbf{2 a}$ & $\mathbf{2 b}$ & $\mathbf{3 a}$ & $\mathbf{3 b}$ \\
\hline BLA & gas & -0.00127 & -0.00354 & 0.000193 & 0.00715 & -0.00984 & -0.01346 \\
& DMF & -0.01566 & -0.0198 & -0.01536 & -0.00897 & -0.02639 & -0.03313 \\
& EA & -0.01236 & -0.01593 & -0.01168 & -0.00496 & -0.0224 & -0.02856 \\
BOA & gas & -0.67557 & -0.71854 & -0.73283 & -0.77109 & -0.68647 & -0.72108 \\
& DMF & -0.66529 & -0.70556 & -0.71771 & -0.75622 & -0.67387 & -0.70846 \\
& EA & -0.66715 & -0.70805 & -0.72069 & -0.75959 & -0.67397 & -0.71079 \\
\hline
\end{tabular}
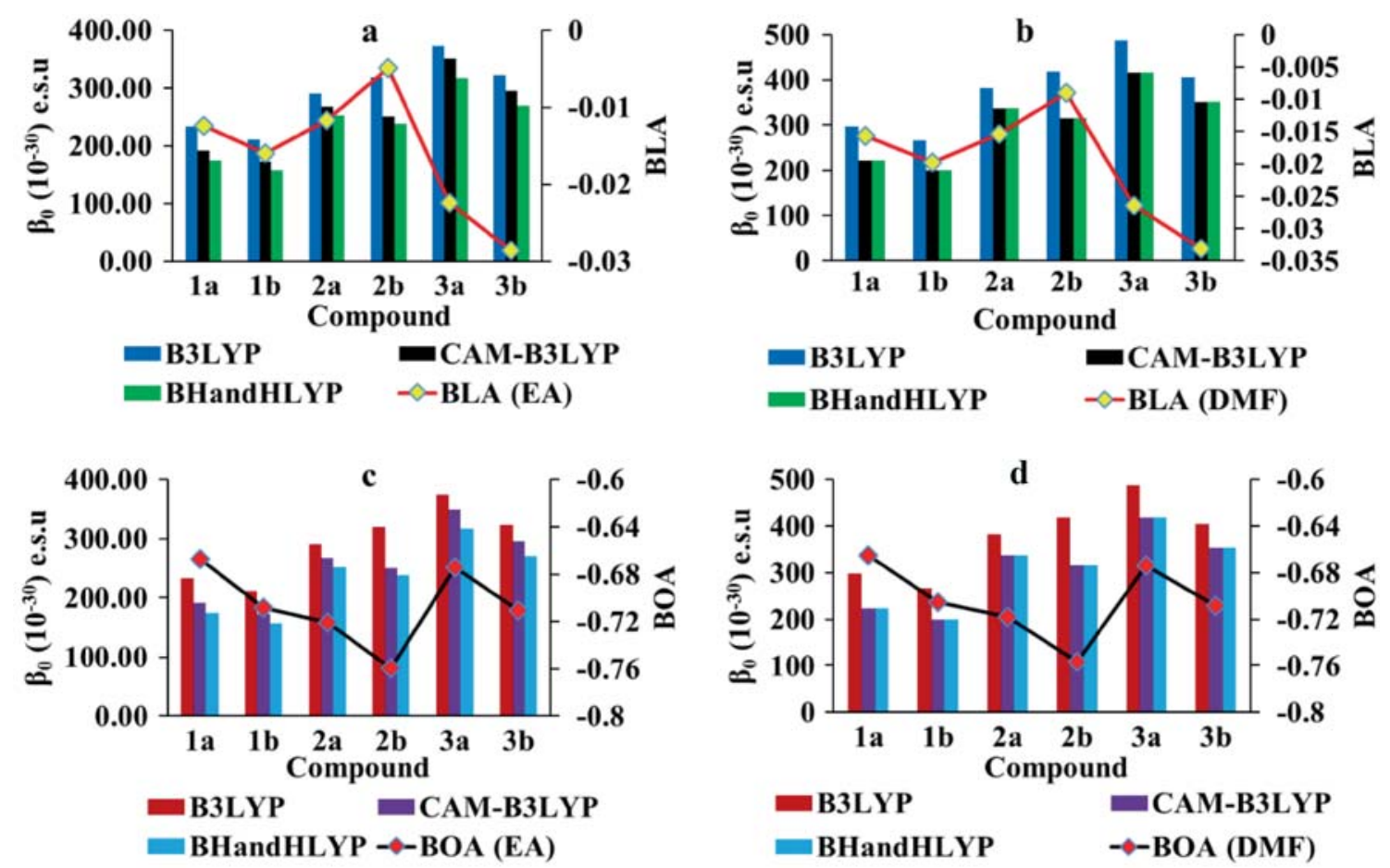

Figure 7. BLA, BOA correlation with $\beta_{0}\left(10^{-30}\right)$ in EA and DMF by B3LYP, CAM-B3LYP and BHandLYP functionals.

$$
\omega^{-}=\frac{\left(E_{\text {НОМO }}\right)^{2}}{2\left(E_{L U M O}-E_{\text {HOMO }}\right)}
$$

both solvents trend of $\mu, \eta, \omega$ and $\omega^{ \pm}$is same (Tables 6 and 7). A good electrophile is indicated by high $\mu$ and $\omega$ and low $\eta$ value. Therefore it can be conclude that the compounds of series a i.e., 1a, 2a and 3a possess good electrophilic character. The plot of inverse of chemical hardness and hyperpolarizability (Figure 6) shows linear correlation it indicates, as first order hyperpolarizability increases from compound $\mathbf{1 a}$ to $\mathbf{3 b}$, molecular stability decrease.

\subsection{Bond length alteration (BLA) and bond order alteration (BOA)}

Bond length alteration(BLA) is the difference between the average carbon-carbon single and double bond lengths in the polymethine backbone, and bond order alteration(BOA) is the difference between the average bond orders of the same two sets of bonds. ${ }^{26}$ These two parameters are important to understand structural features of the compounds. D- $\pi$-A compounds shows neutral and zwitterionic form during the electronic transitions $\left(S_{0}-S_{1}\right)$. Neutral polyene, cyanine and charge separated polyene forms are indicated by positive, zero and negative BLA respectively. Table 8 shows that the BLA values are negative, it indicates the existence of the charge separated form. The trend found in BLA correlated well with the vertical excitations. In series $\mathbf{1}$ and $\mathbf{3}$, a decrease in BLA was observed. In series $\mathbf{2}$, BLA increased as vertical excitation increased from $\mathbf{2 a}$ to $\mathbf{2 b}$. BLA, BOA values show linear correlation with the first order hyperpolarizability $\beta_{0}$ (Figure 7). BLA, $\mathrm{BOA}$ values in DMF and ethylacetate obtained for 1a, 

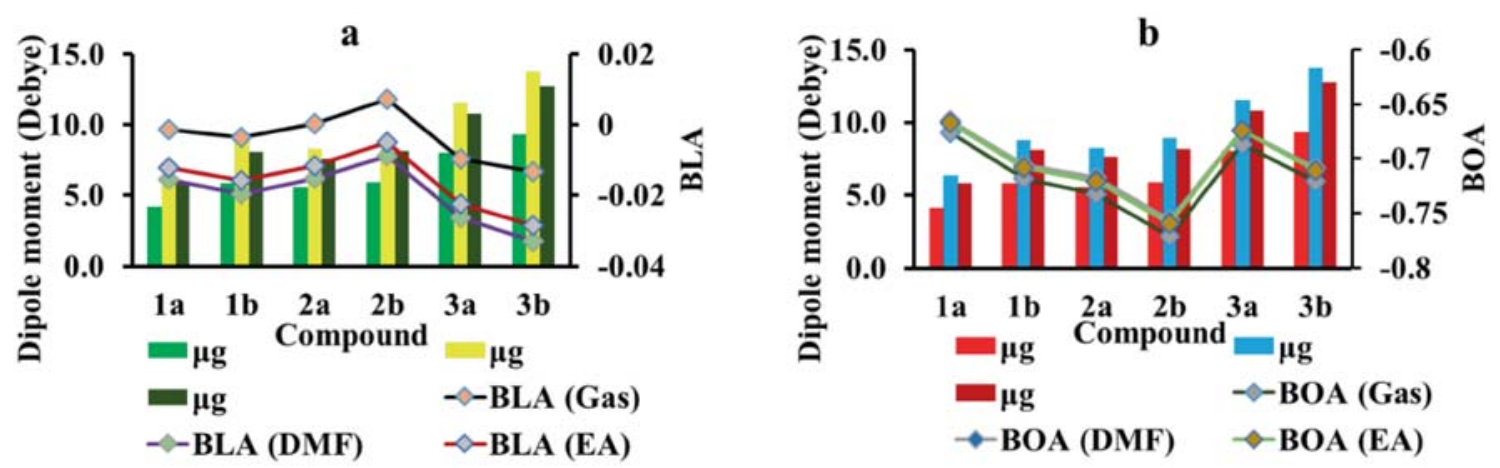

Figure 8. a) BLA, and b) BOA correlation with ground state dipole moment in gas, EA and DMF.
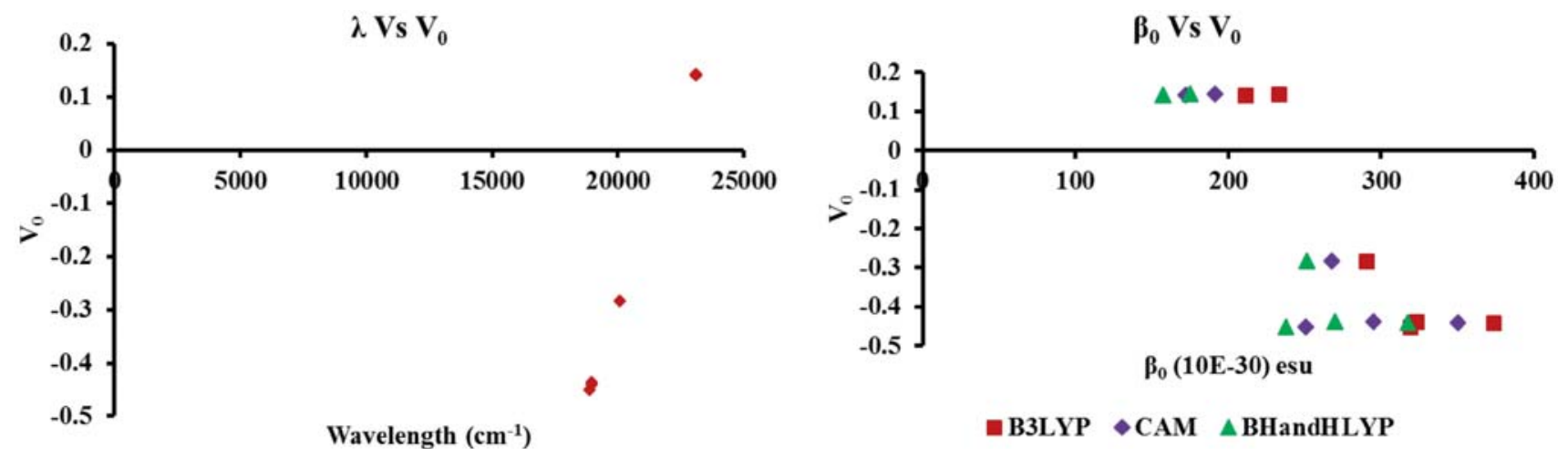

Figure 9. Plot of wavelength and potential energy in DMF and EA.

$\mathbf{2 a}, \mathbf{3 a}, \mathbf{1 b}, \mathbf{2 b}$ and $\mathbf{3 b}$ are summarized in Table 8. BLA increases as the ground state dipole moment of the compounds increases. Increased ground state dipole moment indicate more polarization of the molecules in ground state, therefore charge separated form is more dominating and it supports the negative BLA and $\mathrm{BOA}^{30}$ (Figure 8).

\subsection{Absorption and hyperpolarizability correlation} with the amplitude of the sine shaped potential along the conjugation chain

Hans Kuhn put forth a quantum mechanical theory of light absorption of organic dyes in $1949^{31}$ based on a particle in a box model particularly for the conjugated system which says that the maximum wavelength of absorption $\lambda_{1}$ is expressed as,

$\lambda_{1}=\left(\frac{8 m c}{h}\right)\left(\frac{L^{2}}{[N+1]}\right)$

Where, $m, c$ and $h$ are mass of the electron, velocity of the light and Planck's constant respectively. $L$ is the length of the polyenic chain and $N$ is the number of $\pi$ electrons. The perturbation potential in asymmetrical dyes, when $\pi$ electrons are considered placed in one dimensional potential having a sine curve periodicity is related the absorption, $\lambda_{1}$ expressed as,

$$
\lambda_{1}=\frac{1}{\frac{V_{0}}{h c}\left(1-\frac{1}{N}\right)+\frac{h}{8 m c}\left(\frac{N+1}{L^{2}}\right)}
$$

Where, $V_{0}$ is the amplitude of the sine shaped potential along the chain. $V_{0}$ calculated from eq. (13) and summarized in Table S9 (Supplementary Information). This perturbation potential leading to charge asymmetry within the conjugated system is responsible for the enhancement of NLO response of the molecules. We calculated the potential across the donor carbon $\mathrm{C}_{18}$ and the acceptor carbon $\mathrm{C}_{7}$ (Figure S2, Supplementary Information).

Figure 9 clearly indicates that a decrease in the sinusoidal perturbation potential across the conjugating length decreases the absorption energy decreases (red shift). The static hyperpolarizability decrease as the intramolecular perturbation potential increases (Figure 9). 


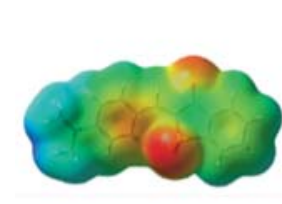

$1 a$

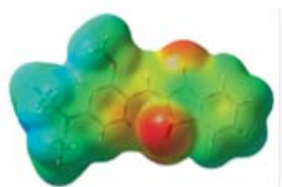

1b

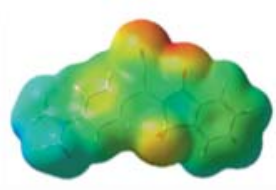

$2 a$

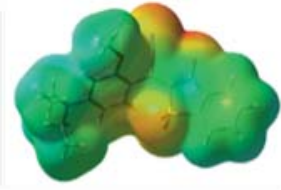

2b

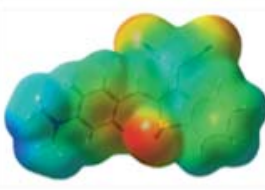

3a

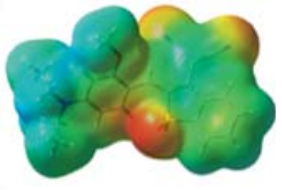

$3 \mathbf{b}$

Figure 10. Molecular Electrostatic Potential (MEP) plots for the compounds 1a-3b in DMF.

\subsection{Molecular electrostatic potential (MEP)}

As studied compounds consist of donor and acceptor, MEP plots were studied to show electrophilic and nucleophilic sites in chromophores which augments charge separation. Charge separation arising out of charge transfer is an important requirement for a molecule to show NLO properties. The red and yellow color indicates the electrophilic reactivity and blue and green color indicates nucleophilic reactivity in the molecule. In series $\mathbf{1}$ and $\mathbf{2}$ the donors $\mathrm{N}, \mathrm{N}$-dimethylaniline and 3-methoxy N,N-diethylaniline show nucleophilic reactivity and acceptor cyano, sulphone and carbonyl group shows electrophilic reactivity (Figure 10). Molecular electrostatic potential observed for the compounds are $\pm 0.008793, \pm 0.00916, \pm 0.102, \pm 0.106, \pm 0.008497$ and \pm 0.008694 for the compounds $\mathbf{1 a}, \mathbf{1 b}, \mathbf{2 a}, \mathbf{2 b}, \mathbf{3 a}$ and $\mathbf{3 b}$ respectively.

\section{Conclusions}

In conclusion we have studied six D- $\pi$-A compounds optimized geometry, vertical excitation energies, Frontier molecular orbitals, HOMO-LUMO, BLA, BOA and NLO properties in ethylacetate and N,N-Dimethylformamide by DFT and TD-DFT. The optimized geometries of the compounds show planarity in the ground state, and in the excited state donor and acceptor groups are orthogonal to each other. Vertical excitation of the compounds were found in good agreement with the experimental absorption. HOMO-LUMO levels were found dependent on nature of donor and acceptor. Donor acceptor strength, dipole moment, BLA and BOA influences the hyperpolarizability. Hyperpolarizability is found more in B3LYP functional than CAM-B3LYP and BHandHLYP functional. Overall hyperpolarizability trend was found as series $\mathbf{3}>\mathbf{2}>$ $\mathbf{1}$ and type $\mathbf{a}>\mathbf{b}$. MEP plots indicate $\mathrm{CO}_{2}, \mathrm{SO}_{2}$ and $\mathrm{CN}$ groups show strong electrophilic reactivity in the molecules. B3LYP functional shows the largest values of polarizability, first and second order hyperpolarizability and intrinsic hyperpolarizability. Comparative study
NLO properties of the compounds $\mathbf{1 a}-\mathbf{3 b}$ shows that $\mathbf{3 a}$ could be a best NLO material.

\section{Supplementary Information (SI)}

This article contains Supplementary Information, which contains selected bond lengths, bond angles and dihedral angles of compounds $\mathbf{2 a}, \mathbf{2} \mathbf{b}, \mathbf{3 a}$ and $\mathbf{3 b}$. Polarizability values in DMF, intrinsic hyperpolarizability in EA and DMF and amplitude of potential are also listed. Supplementary Information is available at www.ias.ac.in/chemsci.

\section{Acknowledgements}

Author Archana A. Bhagwat is thankful to UGC for providing financial assistance.

\section{References}

1. Rao Y L and Wang S 2011 Four-coordinate organoboron compounds with a $\pi$-conjugated chelate ligand for optoelectronic applications Inorg. Chem. 5012263

2. Entwistle C D and Marder T B 2004 Applications of three-coordinate organoboron compounds and polymers in optoelectronics Chem. Mater. 164574

3. Gieseking R L, Mukhopadhyay S, Risko C and Marder S R 2014 Design of polymethine dyes for all-optical switching applications: guidance from theoretical and computational studies Adv. Mater. 2668

4. Polander L E, Pandey L, Barlow S, Tiwari S P, Risko C, Kippelen B, Brédas J-L and Marder S R 2011 Benzothiadiazole-dithienopyrrole donor- acceptordonor and acceptor-donor-acceptor triads: synthesis and optical, electrochemical, and charge-transport properties J. Phys. Chem. C 11523149

5. Rana M, Singla N, Chatterjee A and Shukla A 2016 Investigation of nonlinear optical (NLO) properties by charge transfer contributions of amine functionalized tetraphenylethylene Opt. Mater. 6280

6. Wang X, Mi Y, Wang D, He W, Cao H and Yang H 2016 Third-order nonlinear optical properties of a novel series of azobenzene liquid crystal derivatives Mol. Cryst. Liq. Cryst. 6301

7. Breitung E M, Shu C-F and Mcmahon R J 2000 Thiazole and thiophene analogues of donor - acceptor stilbenes: Molecular hyperpolarizabilities and structure - property relationships J. Am. Chem. Soc. 1221154

8. Meshulam G, Berkovic G and Kotler Z 2000 Electric field induced second harmonic generation with and without fringes Rev. Sci. Instrum. $\mathbf{7 1} 3490$ 
9. Abdel-Halim H M 2003 Electric field induced second harmonic generation: second and third-order hyperpolarizabilities of 4-amino-4/-nitrodiphenyl sulfoxide $J$. Chem. Phys. 119484

10. Hsu C-C, Huang T-H, Zang Y-L, Lin J-L, Cheng Y-Y, Lin $\mathrm{J}$ T, Wu H H, Wang C H, Kuo C-T and Chen C-H 1996 Hyperpolarizabilities of the m-substituent phenyl amine based chromophores determined from the hyperRayleigh scattering and two photon absorption induced fluorescence J. Appl. Phys. 805996

11. Joulaud C, Mugnier Y, Djanta G, Dubled M, Marty JC, Galez C, Wolf J-P, Bonacina L and Le Dante R 2013 Characterization of the nonlinear optical properties of nanocrystals by hyper Rayleigh Scattering $J$. Nanobiotechnol. 11 S8

12. Lanke S K and Sekar N 2016 Coumarin push-pull NL ophores with red emission: solvatochromic and theoretical approach J. Fluoresc. 26949

13. Krawczyk P 2010 DFT study of linear and nonlinear optical properties of donor-acceptor substituted stilbenes, azobenzenes and benzilideneanilines J. Mol. Model. 16 659

14. Tang G, Zhao J, Jiang Z, Kou S, Xu J and Wei C 2012 DFT and ab initio study on non-linear optical (NLO) properties of some organic complexes with different conjugate linker and substituent groups Opt. Spectrosc. 113 240

15. Yanai T, Tew D P and Handy N C 2004 A new hybrid exchange-correlation functional using the Coulomb-attenuating method (CAM-B3LYP) Chem. Phys. Lett. 39351

16. Oudar J L and Chemla D S 1977 Hyperpolarizabilities of the nitroanilines and their relations to the excited state dipole moment J. Chem. Phys. 662664

17. Chemla D S, Oudar J L and Jerphagnon J 1975 Origin of the second-order optical susceptibilities of crystalline substituted benzene Phys. Rev. B 124534

18. Thorat K G, Bhakhoa H, Ramasami P and Sekar N 2014 NIR-emitting boradiazaindacene fluorophores TD-DFT studies on electronic structure and photophysical properties J. Fluoresc. 2569

19. Shenoy V U, Pate1 V P and Seshadri S 1989 Disperse dyes derived from 3-Oxo-2,3-dihydrobenzo- [b] thiophene-1,1-dioxide and 3-dicyanomethylene-2,3dihydrobenzo[ b] thiophene-1,1-dioxide Dye. Pigm. 11 37

20. Frisch M J, Trucks G W, Schlegel H B, Scuseria G E, Robb M A, Cheeseman J R and Scalmani G et al., 2010 Gaussian, Inc., Wallingford CT

21. Treutler O and Ahlrichs R 1995 Efficient molecular numerical integration schemes J. Chem. Phys. 102 346

22. Becke A D 1993 Density functional thermochemistry. III. The role of exact exchange J. Chem. Phys. 985648

23. Lee C, Yang W and Parr R G 1988 Development of the Colle-Salvetti correlation-energy formula into a functional of the electron density Phys. Rev. B. 37785

24. Hehre W J 1976 Ab Initio molecular orbital theory Acc. Chem. Res. 9399

25. Bauernschmitt R and Ahlrichs R 1996 Treatment of electronic excitations within the adiabatic approximation of time dependent density functional theory Chem. Phys. Lett. 256454

26. Meyers F, Marder S R, Pierce B M and Brédas J L 1994 Electric field modulated nonlinear optical properties of donor- acceptor polyenes: sum-over-states investigation of the relationship between molecular polarizabilities ( $\alpha, \beta$ and $\gamma$ ) and bond length alternation J. Am. Chem. Soc. 11610703

27. Lytel R, Mossman S, Crowell E and Kuzyk M G 2017 Exact fundamental limits of the first and second hyperpolarizabilities Phys. Rev. Lett. 11973902

28. Zhou J and Kuzyk M G 2008 Intrinsic hyperpolarizabilities as a figure of merit for electro-optic molecules $J$. Phys. Chem. C 1127978

29. Parr R G, Szentpály L V and Liu S 1999 Electrophilicity index J. Am. Chem. Soc. 1211922

30. Bourhill G, Brédas J-L, Cheng L-T, Marder S R, Meyers F, Perry J W and Tiemann B G 1994 Experimental demonstration of the dependence of the first hyperpolarizability of donor-acceptor-substituted polyenes on the ground-state polarization and bond length alternation $J$. Am. Chem. Soc. 1162619

31. Kuhn H 1949 A quantum mechanical theory of light absorption of organic dyes and similar compounds $J$. Chem. Phys. 171198 